\title{
ARTICLE
}

Epidemiology

\section{Comparative performance of lung cancer risk models to define lung screening eligibility in the United Kingdom}

\author{
Hilary A. Robbins $\mathbb{D}^{1}$, Karine Alcala (D) ${ }^{1}$, Anthony J. Swerdlow ${ }^{2}$, Minouk J. Schoemaker ${ }^{2}$, Nick Wareham ${ }^{3}$, Ruth C. Travis ${ }^{4}$, \\ Philip A. J. Crosbie ${ }^{5}$, Matthew Callister ${ }^{6}$, David R. Baldwin ${ }^{7}$, Rebecca Landy ${ }^{8}$ and Mattias Johansson (iD)
}

BACKGROUND: The National Health Service England (NHS) classifies individuals as eligible for lung cancer screening using two risk prediction models, PLCOm2012 and Liverpool Lung Project-v2 (LLPv2). However, no study has compared the performance of lung cancer risk models in the UK.

METHODS: We analysed current and former smokers aged $40-80$ years in the UK Biobank $(N=217,199)$, EPIC-UK $(N=30,813)$, and Generations Study $(N=25,777)$. We quantified model calibration (ratio of expected to observed cases, E/O) and discrimination (AUC).

RESULTS: Risk discrimination in UK Biobank was best for the Lung Cancer Death Risk Assessment Tool (LCDRAT, AUC $=0.82,95 \%$ $\mathrm{Cl}=0.81-0.84)$, followed by the LCRAT (AUC $=0.81,95 \% \mathrm{Cl}=0.79-0.82)$ and the Bach model $(\mathrm{AUC}=0.80,95 \% \mathrm{Cl}=0.79-0.81)$. Results were similar in EPIC-UK and the Generations Study. All models overestimated risk in all cohorts, with E/O in UK Biobank ranging from 1.20 for LLPv3 $(95 \% \mathrm{Cl}=1.14-1.27)$ to 2.16 for LLPv2 $(95 \% \mathrm{Cl}=2.05-2.28)$. Overestimation increased with area-level socioeconomic status. In the combined cohorts, USPSTF 2013 criteria classified $50.7 \%$ of future cases as screening eligible. The LCDRAT and LCRAT identified 60.9\%, followed by PLCOm2012 (58.3\%), Bach (58.0\%), LLPV3 (56.6\%), and LLPV2 (53.7\%).

CONCLUSION: In UK cohorts, the ability of risk prediction models to classify future lung cancer cases as eligible for screening was best for LCDRAT/LCRAT, very good for PLCOm2012, and lowest for LLPv2. Our results highlight the importance of validating prediction tools in specific countries.

British Journal of Cancer (2021) 124:2026-2034; https://doi.org/10.1038/s41416-021-01278-0

\section{BACKGROUND}

Lung cancer is the leading cause of cancer death worldwide., Two large, randomised trials have now demonstrated that screening by low-dose computed tomography (LDCT) can reduce mortality from lung cancer among people with a heavy smoking history. Lung cancer mortality was reduced by $20 \%$ over 5 years in the USA National Lung Screening Trial (NLST) with 3 annual LDCT screens ${ }^{3}$ and by $24 \%$ (men) and 33\% (women) over 10 years in the Dutch-Belgian NELSON trial with 4 LDCT screens over 5.5 years. ${ }^{4}$

The USA issued a national recommendation for lung screening in 2014. ${ }^{5}$ In the United Kingdom, there have been several successful pilot studies, including the Manchester Lung Health Checks $^{6,7}$ and the Liverpool Healthy Lung Programme. ${ }^{8}$ Compared with the implementation of lung screening in the USA, the UK has often been more successful in terms of overall uptake and engagement of populations with low socioeconomic status (SES), ${ }^{6,9,10}$ and lung cancer detection rates have often exceeded those in the NLST. ${ }^{3,6,11,12}$ Building on this success, the National Health Service (NHS) England is implementing a $£ 70$ million programme of "Targeted Lung Health Checks" in 10 areas with high lung cancer mortality. ${ }^{13,14}$
In the USA, the US Preventive Services Task Force (USPSTF) guidelines use categorical criteria to determine who is eligible for screening. Eligibility by the 2013 guideline required age 55-80 years, at least 30 pack-years smoked, and for former smokers, no more than 15 years since quitting. ${ }^{5}$ The 2020 draft guideline expands eligibility by lowering the age-to-start from 55 to 50 years, and lowering the pack-year threshold from 30 to 20 packyears. ${ }^{15}$ However, secondary analyses of the NLST demonstrated that lung screening may be more efficient and cost-effective when eligibility is based on individual lung cancer risk, estimated using a continuous risk prediction model. ${ }^{16-19}$ Lung screening in the UK was implemented using individual risk-based eligibility from the beginning, and the NHS England protocol specifies that individuals aged 55-74 years can be screened if their lung cancer risk exceeds $1.51 \%$ by the PLCOm 2012 model (6-year risk) or $2.5 \%$ by the Liverpool Lung Project version 2 (LLPV2) model (5-year risk). ${ }^{14}$

The choice of which risk model to use for screening eligibility is important. Poor model discrimination or calibration can reduce the efficiency and cost-effectiveness of screening and even lead to net harm if models select individuals who are unlikely to benefit from screening. Risk models differ in the variables that they

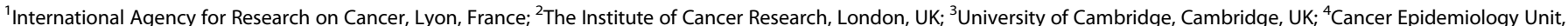

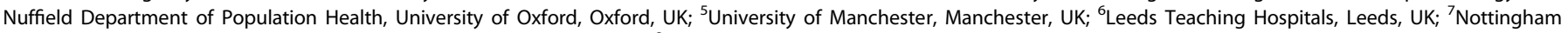

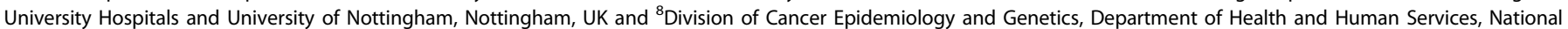
Cancer Institute, National Institutes of Health, Bethesda, MD, USA

Correspondence: Hilary A. Robbins (robbinsh@iarc.fr)

Received: 22 July 2020 Revised: 4 January 2021 Accepted: 13 January 2021

Published online: 12 April 2021 
include; for example, the LLP/LLPv2/LLP model version 3 (LLPV3) models include only one measure of smoking (duration), whereas the Lung Cancer Death Risk Assessment Tool (LCDRAT) includes smoking duration, pack-years, quit-years, and intensity. ${ }^{18,20}$ Most models, including PLCOm2012 and LCDRAT, were developed using USA data, whereas the LLP/LLPV2/LLPv3 models were developed in the UK. ${ }^{17,18,20}$ Although both PLCOm2012 and LLPV2 have been implemented successfully in screening studies, the absence of outcome data on individuals who were not eligible (and thus not screened) has precluded evaluation of whether either of these is the optimal model. ${ }^{6,11}$ Several models have been evaluated in population cohort studies in the USA, ${ }^{21,22}$ but nonUSA evaluations are scarce, ${ }^{23,24}$ and none include data from UK cohorts.

Here we performed a comparative evaluation of lung cancer risk models to define lung screening eligibility in the UK. We analysed 3 cohort studies to quantify the calibration and discrimination of risk models and then compared their ability to classify future lung cancer cases into a group defined as eligible for screening.

\section{METHODS}

We analysed longitudinal data from the UK Biobank, European Prospective Investigation into Cancer and Nutrition (EPIC)-UK, and Generations Study cohorts. The UK Biobank is a prospective cohort study of 500,000 people aged $40-72$ years at recruitment (2006-2010). ${ }^{25}$ EPIC-UK recruited participants aged 45-74 years in Cambridge and aged $\geq 20$ years in Oxford during 1993-2000. ${ }^{26}$ EPIC-Cambridge used population-based recruitment of patients of general practitioners, while EPIC-Oxford was comprised of both population-based recruitment and a subset targeted at "health conscious" individuals. Finally, the Generations Study recruited 112,000 women aged $\geq 16$ years during 2003-2011, of whom about one-third had a mother, daughter, or sister also participating in the study. ${ }^{27}$ In all cohorts, cancer and death ascertainment relied on registry linkages at minimum, sometimes with additional active follow-up. ${ }^{25-27}$

From all participants in these cohorts, we restricted to those known to be current or former smokers who were aged 40-80 years at enrolment, including 217,199 in UK Biobank, 30,813 in EPIC-UK, and 25,777 in the Generations Study (total $N=273,789$ ). Never smokers and participants with unknown smoking status were excluded. After these restrictions, substantial amounts of missing data were present for some variables in some cohorts, such as $31 \%$ missing smoking intensity (cigarettes per day) in UK Biobank. Missing data were handled using various approaches within the framework of multiple imputation (see Supplement). Among participants who were alive and free of lung cancer at the end of follow-up (i.e. in whom future lung cancer status would be unknown), follow-up time was at least 6 years for all participants in UK Biobank and EPIC-UK and for $88 \%$ in the Generations Study.

We evaluated 8 lung cancer risk models. These included the PLCOm2012 and LLPV2 models, which are proposed for use in selecting screening participants in the NHS protocol. ${ }^{11,14,17,20}$ We also evaluated the Bach model, ${ }^{28}$ the LCDRAT, ${ }^{18}$ the Lung Cancer Risk Assessment Tool (LCRAT), ${ }^{18}$ the original LLP model, ${ }^{20}$ the LLPv3 ${ }^{29}$ and the Hoggart model. ${ }^{30}$ Each of these models is either a USA-based model that performs well in USA data (Bach, LCDRAT, LCRAT, PLCOm2012) ${ }^{21}$ or a European model whose performance in European data is unknown (LLP, LLPv2, LLPv3, Hoggart). Risk thresholds above which screening can be offered have been proposed for LCRAT and LCDRAT, ${ }^{19,31}$ and LLPv3, ${ }^{29}$ in addition to PLCOm2012 and LLPV2.

Risk estimates for the LCRAT (5-year time horizon), LCDRAT (5-year), and Hoggart (1-year) models were generated using the Icmodels package in R. ${ }^{32}$ Estimates for the Bach model used code adapted from Icmodels to reduce the time horizon to 5 years. Estimates for PLCOm2012 (6-year time horizon), LLPV3 (5-year),
LLPv2 (5-year), and LLP (5-year) were calculated directly. ${ }^{17,20} \mathrm{We}$ present results for two models in Supplementary Table 1 and do not include them in discussions below, due to redundancy with LLPv2/LLPv3 (LLP) and very high overestimation of risk (Hoggart). We present results for LLPV2 in the main manuscript, even though it may be eventually replaced by LLPV3, because LLPV2 is listed in the NHS England protocol.

We calculated calibration as the ratio of expected to observed $(E / O)$ lung cancer cases or deaths, overall and in subgroups. We quantified discrimination using the area under the receiveroperating curve (AUC) statistic. The $95 \%$ confidence intervals (Cls) for calibration and discrimination statistics account for within and between imputation variance. ${ }^{21}$

\section{RESULTS}

Among the 217,199 current or former smokers aged $40-80$ years in UK Biobank, 1265 lung cancer cases were diagnosed within 5 years of enrolment, and 700 lung cancer deaths occurred in this period (Table 1). In EPIC-UK, 156 lung cancers and 100 lung cancer deaths occurred over 5 years among 30,813 participants, and in the Generations Study, 53 lung cancers and 26 lung cancer deaths occurred over 5 years among 25,777 participants. Distributions of demographic and smoking variables differed across cohorts.

Calibration estimates for all risk models were $>1$ in all cohorts, indicating that the models predicted more lung cancer cases (or for LCDRAT, lung cancer deaths) than were observed over the time period specified by the model (Fig. 1). The extent of overestimation of risks (and therefore poorest calibration) was the highest in the Generations Study and the lowest in UK Biobank for each model. Across the risk models, in UK Biobank, LLPv3 was best calibrated $(E / O=1.20,95 \% \quad C l=1.14-1.27)$, followed by PLCOm2012 $(E / O=1.30,95 \% \mathrm{Cl} 1.23-1.36)$, the Bach model $(E / O=1.39, \quad 95 \% \mathrm{Cl} 1.31-1.47), \quad$ LCRAT $(E / O=1.44,95 \% \mathrm{Cl}$ $1.36-1.52)$, and LCDRAT $(E / O=1.52,95 \% \mathrm{Cl} 1.41-1.64)$. Overestimation was the highest for LLPv2 $(E / O=2.16,95 \% \mathrm{Cl}$ 2.05-2.28). The order in which models were ranked in EPIC-UK and the Generations Study was similar to UK Biobank, but E/O statistics were higher.

Differences in discrimination estimates (AUCs) across models were modest. In UK Biobank, discrimination was the highest for LCDRAT (AUC $=0.82,95 \% \mathrm{Cl} 0.81-0.84$ ) and the lowest for LLPV2 (AUC $=0.77,95 \% \mathrm{Cl}$ 0.76-0.78) (Fig. 2). However, this ordering differed in EPIC-UK, ranging from AUC $=0.84$ for the Bach model (95\% Cl 0.81-0.87) to 0.81 for PLCOm2012 (95\% Cl 0.77-0.85). Discrimination in the Generations Study ranged from AUC $=0.84$ for LCDRAT (95\% Cl $0.77-0.90)$ to 0.78 for PLCOm2012 (95\% Cl $0.71-0.85)$

To further investigate model overestimation, we calculated $E / O$ estimates stratified by demographic and smoking characteristics in UK Biobank (Table 2). Analogous estimates for discrimination (stratified AUCs) are presented in Supplementary Table 2. For all models, there was a strong positive relationship between model overestimation and SES, which was measured by the area-level Townsend deprivation index. For example, for LCDRAT, E/O statistics across SES quartiles were 2.03 (highest SES), 1.95, 1.61, and 1.26 (lowest SES). Patterns for other characteristics differed across models, though frequent patterns included more overestimation in men than in women, in former smokers than in current smokers, and at the extremes of age (40s and 70s). When stratifying by quintiles of predicted risk (Supplementary Fig. 1), PLCOm2012 substantially underestimated risk in the lowest-risk quintile while modestly overestimating risk in the upper categories. Overestimation tended to be higher at higher risks for LLPv2, LLPv3, and Bach, while it was higher at lower risks for LCDRAT and LCRAT.

Table 3 considers the hypothetical impact of using each risk model to determine who is screening eligible in the combined 
Table 1. Characteristics of current and former smokers in UK Biobank, EPIC-UK, and the Generations Study.

\begin{tabular}{|c|c|c|c|}
\hline Characteristic & UK Biobank & EPIC-UK & Generations Study \\
\hline \multicolumn{4}{|l|}{ Lung cancer cases } \\
\hline Within 2 years of enrolment & 420 & 58 & 16 \\
\hline Within 5 years of enrolment & 1265 & 156 & 53 \\
\hline Within 6 years of enrolment & 1461 & 196 & 68 \\
\hline Within 5 years of enrolment & 700 & 100 & 26 \\
\hline Eligible by USPSTF 2013 criteria $^{a}$ & $26,644(12.3 \%)$ & $2484(8.1 \%)$ & $1110(4.3 \%)$ \\
\hline Eligible by USPSTF 2020 criteria $^{a}$ & $45,065(20.7 \%)$ & $5179(16.8 \%)$ & $2994(11.6 \%)$ \\
\hline \multicolumn{4}{|l|}{ Lung cancer cases within 5 years of enrolment } \\
\hline Among USPSTF 2013 eligible individuals ${ }^{a}$ & $616(48.7 \%)$ & $59(37.8 \%)$ & $17(32.1 \%)$ \\
\hline $50-54$ & $30,633(14.1 \%)$ & $5277(17.1 \%)$ & $4773(18.5 \%)$ \\
\hline $55-59$ & $39,632(18.2 \%)$ & $4302(14.0 \%)$ & $5417(21.0 \%)$ \\
\hline $60-64$ & $56,626(26.1 \%)$ & $3813(12.4 \%)$ & $4481(17.4 \%)$ \\
\hline $65-69$ & 44,389 (20.4\%) & $3877(12.6 \%)$ & 2067 (8.0\%) \\
\hline $70-74$ & $1084(0.5 \%)$ & $3268(10.6 \%)$ & 799 (3.1\%) \\
\hline $75-80$ & $0(0.0 \%)$ & $986(3.2 \%)$ & $301(1.2 \%)$ \\
\hline \multicolumn{4}{|l|}{ Sex } \\
\hline Male & $108,043(49.7 \%)$ & $13,191(42.8 \%)$ & $0(0.0 \%)$ \\
\hline Female & $109,156(50.3 \%)$ & $17,622(57.2 \%)$ & $25,777(100.0 \%)$ \\
\hline \multicolumn{4}{|l|}{ Smoking status } \\
\hline \multicolumn{4}{|l|}{ Cigarettes smoked per day } \\
\hline$\leq 10$ & $43,870(20.2 \%)$ & $9845(32.0 \%)$ & $11,223(43.5 \%)$ \\
\hline $11-19$ & $31,488(14.5 \%)$ & 8249 (26.8\%) & $7278(28.2 \%)$ \\
\hline $20-29$ & $55,168(25.4 \%)$ & $5427(17.6 \%)$ & $2905(11.3 \%)$ \\
\hline $30-39$ & $11,468(5.3 \%)$ & $711(2.3 \%)$ & $408(1.6 \%)$ \\
\hline$\geq 40$ & 8619 (4.0\%) & $435(1.4 \%)$ & $125(0.5 \%)$ \\
\hline Unknown & $66,586(30.7 \%)$ & $6146(19.9 \%)$ & $3838(14.9 \%)$ \\
\hline \multicolumn{4}{|l|}{ Educational level } \\
\hline Less than secondary & $44,950(20.7 \%)$ & $6248(20.3 \%)$ & $6(0.0 \%)$ \\
\hline Secondary degree & $58,098(26.7 \%)$ & $12,031(39.0 \%)$ & 10,947 (42.5\%) \\
\hline Some post-secondary training & $33,688(15.5 \%)$ & NA & NA \\
\hline Some university & $16,126(7.4 \%)$ & NA & 7996 (31.0\%) \\
\hline University graduate & $60,363(27.8 \%)$ & $6369(20.7 \%)$ & $6223(24.1 \%)$ \\
\hline Unknown & 3974 (1.8\%) & $6165(20.0 \%)$ & $605(2.3 \%)$ \\
\hline \multicolumn{4}{|l|}{ Body mass index } \\
\hline Underweight & $1100(0.5 \%)$ & $356(1.2 \%)$ & $167(0.6 \%)$ \\
\hline Normal weight & $64,430(29.7 \%)$ & $14,776(48.0 \%)$ & $12,220(47.4 \%)$ \\
\hline Overweight & $93,806(43.2 \%)$ & 11,927 (38.7\%) & 8658 (33.6\%) \\
\hline Obese & $56,651(26.1 \%)$ & $3754(12.2 \%)$ & $4233(16.4 \%)$ \\
\hline Unknown & $1212(0.6 \%)$ & $0(0.0 \%)$ & 499 (1.9\%) \\
\hline
\end{tabular}




\begin{tabular}{|llll|}
\hline Table 1. continued & & & \\
\hline Characteristic & UK Biobank & EPIC-UK & Generations Study \\
\hline Personal history of cancer & $10,651(4.9 \%)$ & $1123(3.6 \%)$ & $1722(6.7 \%)$ \\
First-degree family history of lung cancer & $18,039(8.3 \%)$ & Missing & Missing \\
COPD or emphysema & $4046(1.9 \%)$ & Missing & Missing \\
Prior pneumonia & $1346(0.6 \%)$ & Missing & Missing \\
Asbestos exposure & Missing & $2167(7.0 \%)$ & Missing \\
\hline
\end{tabular}

Table 1 shows data prior to imputation of missing data. UK educational categories were mapped to USA categories as described in the Supplement. Body mass index categories were defined as follows: $<18.5$ underweight, 18.5-24.9 normal weight, 25-29.9 overweight, and $\geq 30$ obese. "Asbestos exposure" reflects selfreported occupational asbestos exposure.

COPD chronic obstructive pulmonary disease, NA not applicable.

${ }^{a}$ Eligibility by the US Preventive Services Task Force (USPSTF) 2013 criteria requires age 55-80 years, at least 30 pack-years, and no more than 15 quit-years. Eligibility by the draft USPSTF 2020 criteria requires age 50-80 years, at least 20 pack-years, and no more than 15 quit-years.

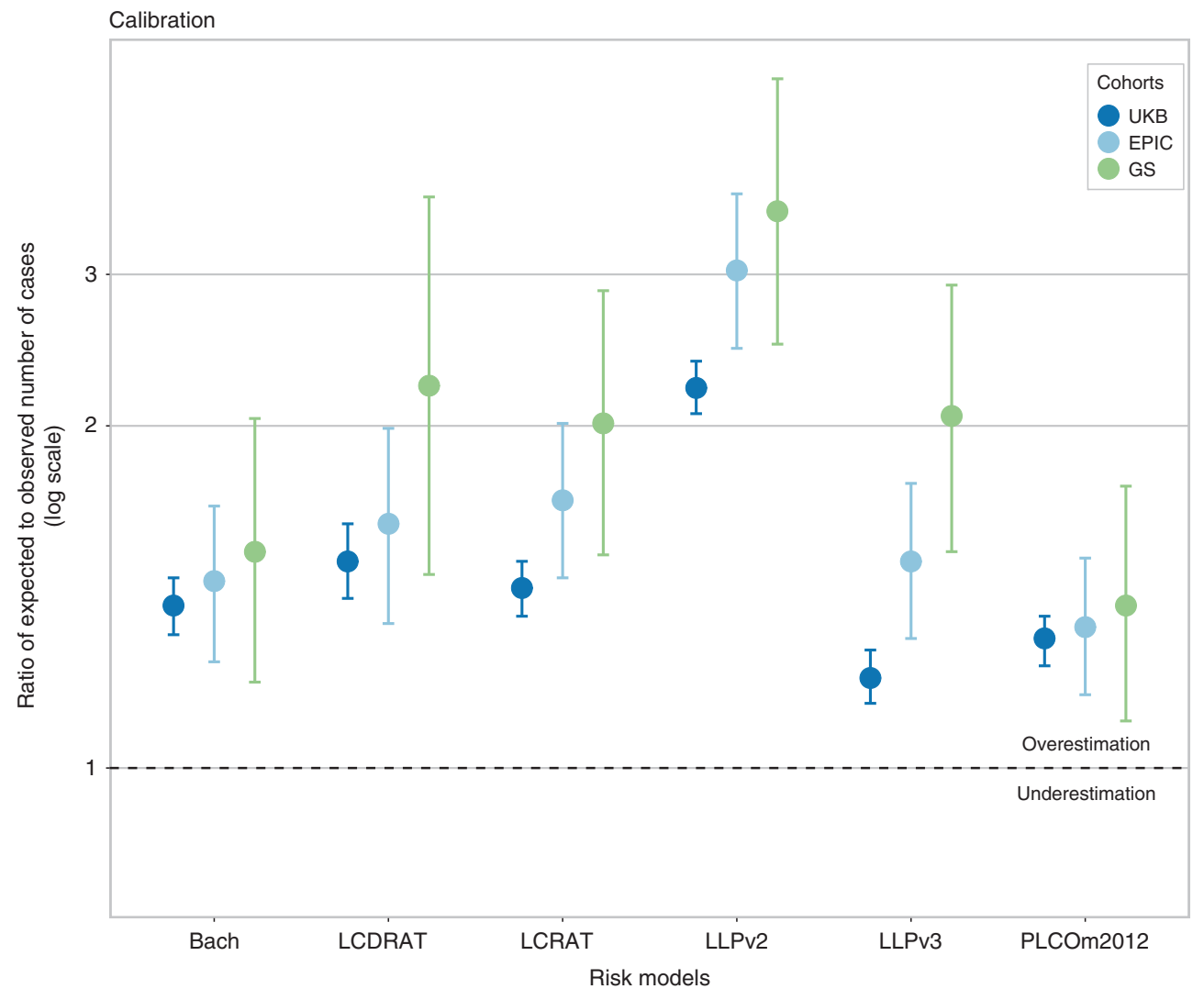

Fig. 1 Calibration of lung cancer risk models in the UK Biobank, EPIC-UK, and Generations Study cohorts, as measured by the ratio of expected to observed cases. UKB UK Biobank, GS Generations Study. Estimates for UK Biobank also appear in Table 2 and Supplementary Table 3.

population of UK Biobank, EPIC-UK, and the Generations Study. In the combined population, after imputing missing data, $15.0 \%$ of individuals were eligible for screening by USPSTF 2013 criteria (age 55-80 years, at least 30 pack-years, no more than 15 quit-years). The thresholds that would screen the same number of individuals using risk-based eligibility were $0.8 \%$ for LCDRAT (5-year lung cancer death risk), 1.4\% for LCRAT (5-year lung cancer risk), 1.5\% for PLCOm2012 (6-year risk), 1.6\% for Bach (5-year risk), 1.3\% for LLPv3 (5-year risk), and 2.3\% for LLPv2 (5year risk).

Using USPSTF 2013 guidelines to define screening eligibility, $50.7 \%$ of future lung cancer cases $(N=747)$ would be classified as eligible for screening (Table 3 ). Similarly, $50.2 \%$ of future lung cancer deaths $(N=415)$ over 5 years would be screening eligible, among which some fraction could be prevented by earlier detection. Applying risk models and the thresholds described above to define the screened population identified higher proportions of future cases: the LCDRAT and LCRAT identified the highest proportion of future cases as screening eligible (each $60.9 \%, N=897)$, followed by PLCOm2012 (58.3\%, $N=859)$, Bach (58.0\%, $N=855), \operatorname{LLPv3}(56.6 \%, N=835)$, and LLPv2 $(53.7 \%, N=$ 791). For lung cancer deaths, the ranking of models was similar, with LCDRAT identifying $63.2 \%$ of lung cancer deaths as screening eligible $(N=522)$, followed by LCRAT $(62.8 \%, N=519)$, Bach $(60.7 \%, N=501)$, PLCOm2012 (59.3\%, $N=490)$, LLPv3 $(59.2 \%, N=$ 489), and LLPV2 (56.3\%, $N=465)$. 


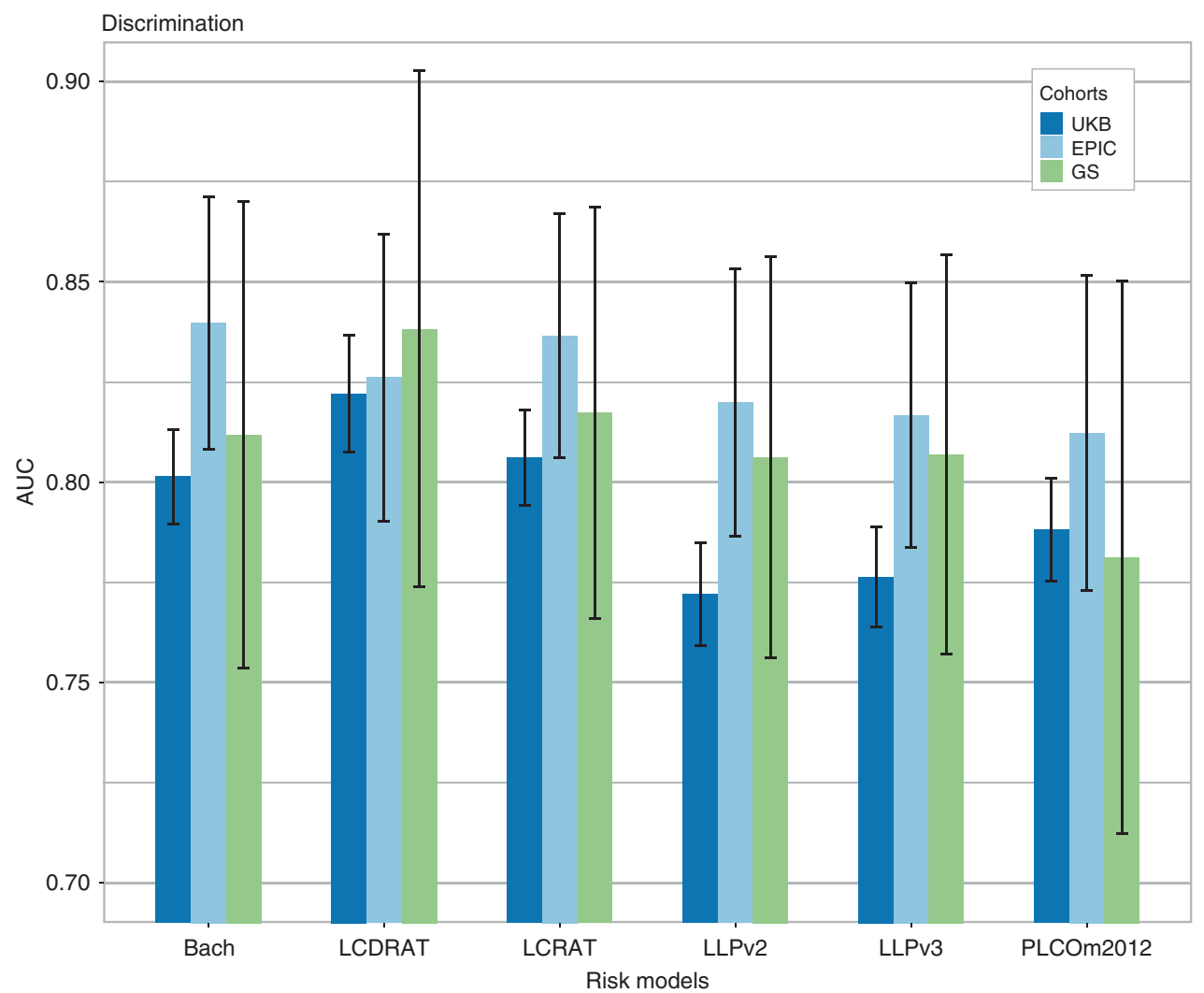

Fig. 2 Discrimination of lung cancer risk models in the UK Biobank, EPIC-UK, and Generations Study cohorts, as measured by the area under the ROC curve (AUC). UKB UK Biobank, GS Generations Study. Estimates for UK Biobank also appear in Supplementary Tables 2 and 3.

Applying USPSTF 2020 criteria (age 50-80 years, at least 20 pack-years, no more than 15 quit-years) yielded lower risk thresholds for screening and higher percentages of cases classified as screening eligible, as expected (Table 3). USPSTF 2020 criteria identified $26.1 \%$ of participants and $66.4 \%$ of future lung cancer cases as screening eligible. If risk models also screened the $26.1 \%$ highest-risk participants, LCDRAT would identify $77.0 \%$ of cases at a threshold of $0.4 \% 5$-year risk $(N=$ 1135 ) followed by Bach $(76.7 \%, N=1131$ at $0.8 \%$ 5-year risk), PLCOm2012 (75.2\%, $N=1109$ at 0.8\% 6-year risk), LCRAT (74.7\%, $N=1101$ at $0.8 \% 5$-year risk), LLPv3 $(70.5 \%, N=1039$ at $0.7 \% 5-$ year risk), and LLPV2 (69.2\%, $N=1020$ at 1.3\% 5-year risk). Results for lung cancer deaths were similar.

We analysed individuals aged $40-80$ years, but the NHS England protocol restricts eligibility to ages $55-74$ years. When we repeated our analysis after restricting to individuals aged 55-74 years in UK Biobank (n.b. there were no participants in UK Biobank aged $>74$ years), AUCs decreased as expected due to the loss in prediction derived from age variation. However, the rank order of AUCs and the calibration results were not affected (Table 4).

Supplementary Table 3 describes the characteristics of lung cancer cases that are not identified as screening eligible (are "missed") by USPSTF 2013, USPSTF 2020, and each risk model at the risk thresholds identified in Table 3. Compared with USPSTF 2013, the cases missed by risk models, while fewer in number, were more commonly former smokers (62\% of cases missed by USPSTF vs. $69-80 \%$ for risk models). They also tended to be slightly younger (median age at baseline 63 years for cases missed by USPSTF vs. 60-61 years for risk models) and slightly more frequently female ( $53 \%$ of cases missed by USPSTF vs. $54-57 \%$ for risk models). Patterns using thresholds based on USPTF 2020 were similar or more pronounced.

\section{DISCUSSION}

Lung cancer screening has the potential to substantially reduce lung cancer mortality among people with a heavy smoking history. In the United Kingdom, the success of the Targeted Lung Health Checks will depend partially on whether the programme can be implemented efficiently and cost-effectively. The protocol currently recommends the use of the PLCOm2012 and LLPV2 risk models to identify screening-eligible individuals. ${ }^{14}$ In this study, we compared the performance of these two models along with others that have performed well in other high-income settings. We found that the LLPV2 model had worst calibration and classified the lowest proportion of future lung cancer cases as eligible for screening. The PLCOm2012 model had better calibration, though all models predicted more cases than were observed. The LCDRAT was able to classify the highest proportion of future lung cancer cases as eligible for screening, with very good performance also observed for the LCRAT, PLCOm2012, and Bach models.

The models evaluated in our study were previously validated in multiple USA cohorts, including the NIH-AARP and CPS-II, ${ }^{21}$ as well as the NLST and PLCO trials. ${ }^{22}$ Taken together, these studies showed good calibration for the Bach model, LCRAT, LCDRAT, and PLCOm2012 but overestimation of risks for the LLP model. In our study, all models overestimated risks; the extent was greatest for LLPV2. For discrimination, prior results in NIH-AARP and CPS-II showed best performance for LCDRAT, followed sequentially by LCRAT, PLCOm2012, Bach, and LLP. ${ }^{21}$ The study analysing PLCO and NLST found higher discrimination for PLCOm2012 and Bach compared with LLP. ${ }^{22}$ The order in which models ranked in our study was similar, with best performance for the LCDRAT, LCRAT, Bach, and PLCOm2012 models. The likely explanation for inferior discrimination of the LLP/LLPV2/LLPv3 models is that they 
Table 2. Calibration estimates for lung cancer risk models in the subgroups of UK Biobank, as measured by the ratio of expected to observed cases.

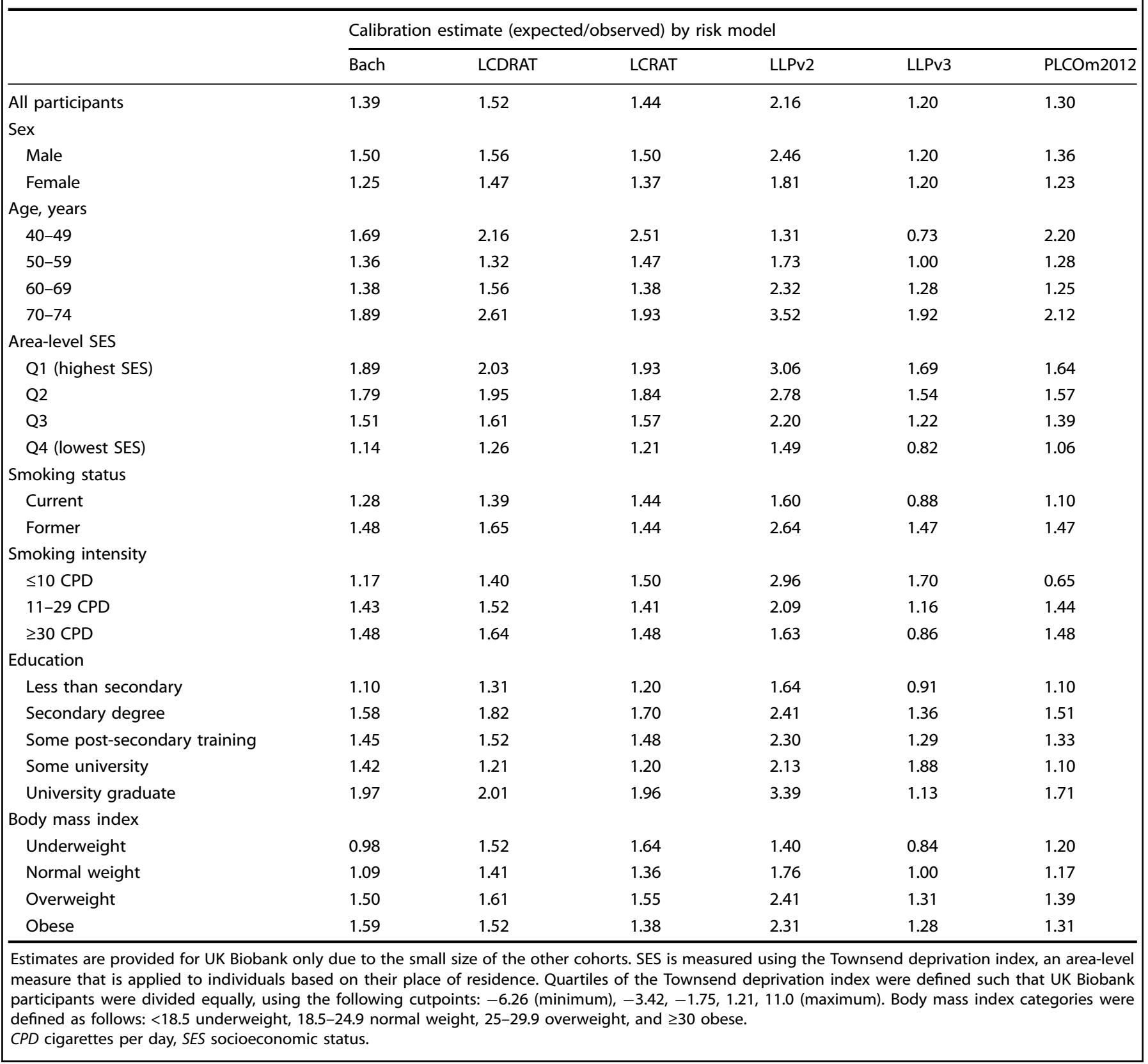

incorporate only smoking duration (omitting intensity and quityears) and use categorical instead of continuous parameterisations of age and smoking. ${ }^{20}$ Overall, the magnitude of AUCs in our study (often exceeding 0.80) was higher than in prior reports (typically ranging from 0.75 to 0.80 ). ${ }^{21,22}$ This is likely caused by a wider age distribution in our analysis, which included more younger individuals.

In UK screening studies, lung cancer detection rates have commonly been higher than in the NLST. ${ }^{3,6,7,11}$ Lung cancer detection over 2 screens was $4.5 \%$ in the Manchester Lung Health Checks, compared with $1.7 \%$ in NLST. ${ }^{3,6,7}$ Detection rates in singlescreen UK studies are commonly approximately $2 \% .^{11,12}$ These observations might have been taken as evidence that USA-based lung cancer risk models would predict too few cases in UK populations, but we found the opposite result. Unlike the screening studies, which commonly comprised individuals living in low-SES communities, ${ }^{6,11,12}$ the research cohorts we analysed have overrepresentation of high-SES individuals and are likely influenced by "healthy volunteer" effects. In UK Biobank, all-cause mortality among 70-74-year-olds is half that in the general population (although the difference in cancer incidence is smaller). ${ }^{33}$ EPIC-Oxford is partially comprised of "health-conscious" individuals. The Generations Study is a volunteer cohort with recruitment based on engagement in a health issue (finding the causes of breast cancer), and cancer incidence is estimated to be $16 \%$ lower than in the general UK population (unpublished data). These influences, taken together with the overrepresentation of high-SES individuals in whom overestimation is highest, suggest that model calibration would be better in the overall UK population of ever-smokers than in the research cohorts we analysed.

There is a troubling consequence to the correlation between risk model overestimation and SES. Considering four individuals with the same true risk of lung cancer, one in each SES quartile, 
Table 3. Performance of lung cancer risk models for defining lung cancer screening eligibility among current and former smokers in the combined UK Biobank, EPIC-UK, and Generations Study cohorts.

\begin{tabular}{|c|c|c|c|}
\hline Risk model & $\begin{array}{l}\text { Threshold to } \\
\text { screen the same } \\
\text { number of } \\
\text { participants as } \\
\text { USPSTF guidelines }\end{array}$ & $\begin{array}{l}\text { Lung cancer } \\
\text { cases eligible } \\
\text { for screening } \\
\text { over } 5 \text { years }\end{array}$ & $\begin{array}{l}\text { Lung cancer } \\
\text { deaths eligible } \\
\text { for screening } \\
\text { over } 5 \text { years }\end{array}$ \\
\hline
\end{tabular}

USPSTF 2013 guidelines (age 55-80 years, at least 30 pack-years, no more than 15 quit-years)

\begin{tabular}{|c|c|c|c|}
\hline Total & NA & $1474(100 \%)$ & $826(100 \%)$ \\
\hline $\begin{array}{l}\text { USPSTF } \\
\text { criteria }\end{array}$ & NA & 747 (50.7\%) & $415(50.2 \%)$ \\
\hline LCDRAT & $0.8 \% 5$-year risk & 897 (60.9\%) & $522(63.2 \%)$ \\
\hline LCRAT & $1.4 \% 5$-year risk & 897 (60.9\%) & 519 (62.8\%) \\
\hline PLCOm2012 & $1.5 \%$ 6-year risk & 859 (58.3\%) & 490 (59.3\%) \\
\hline Bach & $1.6 \% 5$-year risk & 855 (58.0\%) & $501(60.7 \%)$ \\
\hline LLPv3 & $1.3 \% 5$-year risk & $835(56.6 \%)$ & 489 (59.2\%) \\
\hline LLPv2 & $2.3 \%$ 5-year risk & 791 (53.7\%) & 465 (56.3\%) \\
\hline
\end{tabular}

USPSTF 2020 guidelines (age 50-80 years, at least 20 pack-years, no more than 15 quit-years)

\begin{tabular}{|c|c|c|c|}
\hline Total & NA & 1474 (100\%) & 826 (100\%) \\
\hline $\begin{array}{l}\text { USPSTF } \\
\text { criteria }\end{array}$ & NA & 979 (66.4\%) & $560(67.8 \%)$ \\
\hline LCDRAT & $0.4 \% 5$-year risk & 1135 (77.0\%) & 655 (79.3\%) \\
\hline LCRAT & $0.8 \% 5$-year risk & 1101 (74.7\%) & 637 (77.1\%) \\
\hline PLCOm2012 & $0.8 \%$ 6-year risk & 1109 (75.2\%) & 629 (76.2\%) \\
\hline Bach & $0.8 \% 5$-year risk & 1131 (76.7\%) & 652 (78.9\%) \\
\hline LLPv3 & $0.7 \%$ 5-year risk & 1039 (70.5\%) & 604 (73.1\%) \\
\hline LLPv2 & $1.3 \% 5$-year risk & 1020 (69.2\%) & 590 (71.4\%) \\
\hline
\end{tabular}

Models are listed in order of their performance for identifying future lung cancer cases (USPSTF 2013). "Risk" refers to lung cancer death risk for LCDRAT and to lung cancer risk for all other models. Results are based on a combined data set, which uses a single imputation for missing data. USPSTF 2013 criteria classify 41,107 (15.0\%) current and former smokers in the 3 cohorts combined as screening eligible, and USPSTF 2020 criteria classify $71,387(26.1 \%)$ as eligible. These percentages differ from Table 1 as they are calculated after imputation, whereas Table 1 omits missing data. NA not applicable.

the individual with the highest SES will have her risk overestimated the most and will be most likely to be classified as eligible for screening. This could exacerbate disparities in lung screening. ${ }^{34}$ Our findings suggest that there are factors related to SES that increase lung cancer risk but are not captured by the variables in risk models or are related to differential measurement error for the variables in risk models. Further, the effects of USA educational and ethnicity categories on lung cancer risk are unlikely to align with these effects in the UK. Any future efforts to develop risk models for use in lung screening in the UK should focus carefully on the role of SES and the accurate estimation of risk within subgroups.

The choice of what risk threshold to use for screening eligibility depends on multiple factors, including the accepted trade-off of benefits and harms and the capacity of the health system. We did not address these issues here, but we did identify thresholds that would classify the same number of individuals as screening eligible as USPSTF criteria. The thresholds selected by this approach, when considering USPSTF 2013 criteria, aligned with those already proposed for the PLCOm2012 (1.5\% in our study vs. $1.51 \%$ in the NHS protocol) and the LLPv2 $(2.3 \%$ in our study vs. $2.5 \%$ in the NHS protocol). ${ }^{14}$ There was a larger difference between the threshold we identified for LCDRAT (0.8\% 5-year lung cancer death risk) and previously proposed thresholds (1.2, 1.33, 1.7\%). ${ }^{19,31}$ Thresholds identified based on USPSTF 2020 criteria, which broadened eligibility substantially, were much lower, and it is not clear whether all individuals meeting these thresholds would have a favourable trade-off of screening benefits and harms.

Important limitations of risk-based eligibility for lung screening are receiving increased recognition. Risk models preferentially select older individuals, reducing life-years gained and costeffectiveness, as well as individuals with comorbidities such as COPD who may have lower screening benefits. ${ }^{19,35-37}$ To address these issues, a model to define eligibility based on predicted lifeyears gained from screening has been proposed, ${ }^{37}$ which incorporates LCDRAT and an additional prediction model for overall mortality. Important evidence for the comparative performance of PLCOm2012 and LLPV2 will be provided by the Yorkshire Lung Screening Trial, which is enrolling participants based on eligibility by either model to compare their performance directly. ${ }^{38}$ The trial is also collecting sufficient information to validate the LCRAT, LCDRAT, and Bach models retrospectively.

Our study has important limitations that result from its approach of analysing cohort data. Our findings cannot be assumed to be nationally representative for the UK, though the rank-order performance of lung cancer risk prediction models is likely generalisable. There was also a substantial amount of missing data for key smoking variables, which we handled by multiple imputation. For comparison, we calculated E/O statistics and AUCs using the subset of individuals in UK Biobank who had complete data on required variables (63\% of the cohort). The degree of overestimation was reduced for all models (Supplementary Fig. 2), while AUCs were not affected (Supplementary Fig. 3). By contrast, our approach of analysing multiple cohorts is a strength, because it allows for evaluating whether results are consistent across studies. Inclusion of the Generations Study, despite its small size, is important due to the underrepresentation of women in the European screening trial literature. Women in the Generations Study had lower smoking intensity and longer periods of cessation compared with participants in the other studies, highlighting potential equity issues around screening eligibility.

The question of which risk model optimally defines screening eligibility is somewhat distinct from the question of which model can most practically be implemented. Web-based tools are available for some risk models, including the Risk-based NLST Outcomes Tool for LCDRAT and LCRAT ${ }^{39}$ and MyLungRisk for LLPv2 ${ }^{40}$ and a spreadsheet tool is available for PLCOm2012. ${ }^{41}$ It is possible to integrate these tools into electronic medical records to facilitate calculations, but input information would first need to be verified with the patient. A practical solution may be to use a simplified model or algorithm applied to electronic medical records to initially identify people who are potentially eligible, followed by a more precise assessment of risk using data collected in person or by phone. The goal to automate further the calculation of lung cancer risk and the classification of individual screening eligibility represents an ongoing challenge.

In conclusion, we analysed the performance of lung cancer risk models in three UK cohorts, including the PLCOm2012 and LLPV2 models that are recommended for use in lung screening by the NHS protocol for Targeted Lung Health Checks. We found that the LLPV2 model had worst calibration and classified the lowest proportion of future lung cancer cases as eligible for screening. The LLPV3 model had best calibration (but poor discrimination), while the LCDRAT was best able to identify individuals at high risk of lung cancer. All models strongly over-predicted risk in groups with high SES, raising concerns about exacerbation of disparities in lung cancer screening. Taken together, our results suggest potential revisions to the list of models endorsed by the NHS lung 
Table 4. Calibration and discrimination estimates for lung cancer risk models in UK Biobank current and former smoking participants, restricted to ages $55-74$ years.

\begin{tabular}{lllllll}
\hline & \multicolumn{2}{l}{ Lung cancer risk model } & & & \\
\cline { 2 - 6 } & Bach & LCDRAT & LCRAT & LLPv2 & LLPv3 \\
\hline Calibration $(E / O)$ & & & & & \\
Among age 40-74 years & $1.39(1.31-1.47)$ & $1.52(1.41-1.64)$ & $1.44(1.36-1.52)$ & $2.16(2.05-2.28)$ & $1.20(1.14-1.27)$ & $1.30(1.23-1.36)$ \\
Among age 55-74 years & $1.38(1.30-1.47)$ & $1.52(1.41-1.64)$ & $1.40(1.32-1.48)$ & $2.25(2.13-2.39)$ & $1.25(1.18-1.33)$ & $1.26(1.19-1.33)$ \\
Discrimination (AUC) & & & & & & \\
Among age 40-74 years & $0.80(0.79-0.81)$ & $0.82(0.81-0.84)$ & $0.81(0.79-0.82)$ & $0.77(0.76-0.78)$ & $0.78(0.76-0.79)$ & $0.79(0.78-0.80)$ \\
Among age 55-74 years & $0.76(0.75-0.78)$ & $0.79(0.77-0.81)$ & $0.77(0.76-0.79)$ & $0.73(0.72-0.75)$ & $0.74(0.73-0.75)$ & $0.75(0.74-0.77)$ \\
\hline
\end{tabular}

The $E / O$ statistic is the ratio of cases expected (predicted by the model) to the cases observed. AUC is the area under the receiver operating curve. The estimates for ages 40-74 years correspond with the primary estimates presented in Fig. 1 (n.b. there were no participants in UK Biobank aged >74 years).

screening protocol and that further work may be needed to ensure that eligibility for lung cancer screening can be defined equitably in the UK population. More generally, they highlight the importance of carefully validating risk prediction models in specific contexts before they are applied in practice.

\section{DISCLAIMER}

Where authors are identified as personnel of the International Agency for Research on Cancer/World Health Organisation, the authors alone are responsible for the views expressed in this article and they do not necessarily represent the decisions, policy or views of the International Agency for Research on Cancer/World Health Organisation.

\section{ACKNOWLEDGEMENTS}

We thank all study participants, study staff, and the doctors, nurses and other health care staff and data providers who contributed to the UK Biobank, EPIC-UK, and Generations Study. This research has been conducted using the UK Biobank Resource under application number 15825. We thank Dr. Paul Brennan for his feedback on the manuscript.

\section{AUTHOR CONTRIBUTIONS}

H.A.R. and M.J. conceived of the study and its design. A.J.S., M.J.S., N.W., and R.C.T. provided study data. K.A. and H.A.R. completed statistical analyses. H.A.R., K.A., A.J.S., M.J.S., N.W., R.C.T., M.C., R.L., P.A.J.C., D.R.B., and M.J. interpreted the results. H.A.R. drafted the manuscript. H.A.R., K.A., A.J.S., M.J.S., N.W., R.C.T., M.C., R.L., P.A.J.C., D.R.B., and M.J. critically reviewed the draft manuscript and approved the final version.

\section{ADDITIONAL INFORMATION}

Ethical approval and consent to participate The UK Biobank was approved by the North West Multi-Centre Research Ethics Committee. The Generations Study was approved by the South East Multi-Centre Research Ethics Committee. EPIC was approved by the IARC Ethics Committee and by ethics committees at participating EPIC Centres. Cohort participants provided written informed consent prior to participating.

Data availability All analysis codes are available upon request. UK Biobank data are available to researchers with an approved request. Access to data from EPIC and the Generations Study requires an application process; further information is available at epic.iarc.fr and breakthroughgenerations.org.uk.

Competing interests H.A.R., K.A., A.J.S., M.J.S., N.W., R.C.T., M.C., R.L., M.J.: None. P.A.J. C.: consultancy and share options, Everest Detection. D.R.B.: reimbursement for participation on advisory boards, AstraZeneca, and MSD.

Funding information This study was funded in part by the US National Cancer Institute (R03 CA245979 and U19 CA203654) and Cancer Research UK (C18281/ A19169). The Generations Study was funded by Breast Cancer Now and the Institute of Cancer Research. The ICR acknowledges NHS funding to the Royal Marsden/ICR NIHR Biomedical Research Centre. P.A.J.C. is supported by the NIHR Manchester Biomedical Research Centre. M.C. is co-chief investigator of the Yorkshire Lung Screening Trial, which is funded by Yorkshire Cancer Research. R.L. is supported by the Intramural Research Program of the US National Institutes of Health/National Cancer Institute. The funders had no role in the design, analysis, or reporting of the study.

Supplementary information The online version contains supplementary material available at https://doi.org/10.1038/s41416-021-01278-0.

Publisher's note Springer Nature remains neutral with regard to jurisdictional claims in published maps and institutional affiliations.

\section{REFERENCES}

1. Cancer Research UK. Cancer Statistics for the UK. https://www.cancerresearchuk. org/health-professional/cancer-statistics-for-the-uk (2020).

2. Bray, F., Ferlay, J., Soerjomataram, I., Siegel, R. L., Torre, L. A. \& Jemal, A. Global cancer statistics 2018: GLOBOCAN estimates of incidence and mortality worldwide for 36 cancers in 185 countries. CA Cancer J. Clin. 68, 394-424 (2018).

3. National Lung Screening Trial Research Team, Aberle, D. R., Adams, A. M., Berg, C. D., Black, W. C., Clapp, J. D. et al. Reduced lung-cancer mortality with low-dose computed tomographic screening. N. Engl. J. Med. 365, 395-409 (2011).

4. de Koning, H. J., van der Aalst, C. M., de Jong, P. A., Scholten, E. T., Nackaerts, K., Heuvelmans, M. A. et al. Reduced lung-cancer mortality with volume CT screening in a randomized trial. N. Engl. J. Med. 382, 503-513 (2020).

5. Moyer, V. A. Screening for lung cancer: U.S. Preventive Services Task Force recommendation statement. Ann. Intern. Med. 160, 330-338 (2014).

6. Crosbie, P. A., Balata, H., Evison, M., Atack, M., Bayliss-Brideaux, V., Colligan, D. et al. Implementing lung cancer screening: baseline results from a communitybased "Lung Health Check" pilot in deprived areas of Manchester. Thorax 74, 405-409 (2018).

7. Crosbie, P. A., Balata, H., Evison, M., Atack, M., Bayliss-Brideaux, V., Colligan, D. et al. Second round results from the Manchester "Lung Health Check" communitybased targeted lung cancer screening pilot. Thorax 74, 700-704 (2018).

8. Ghimire, B., Maroni, R., Vulkan, D., Shah, Z., Gaynor, E., Timoney, M. et al. Evaluation of a health service adopting proactive approach to reduce high risk of lung cancer: The Liverpool Healthy Lung Programme. Lung Cancer 134, 66-71 (2019).

9. Jemal, A. \& Fedewa, S. A. Lung cancer screening with low-dose computed tomography in the United States - 2010 to 2015. JAMA Oncol. 3, 1278-1281 (2017).

10. Quaife, S. L., Ruparel, M., Dickson, J. L., Beeken, R. J., McEwen, A., Baldwin, D. R. et al. Lung Screen Uptake Trial (LSUT): randomized controlled clinical trial testing targeted invitation materials. Am. J. Respir. Crit. Care Med. 201, 965-975 (2020).

11. Field, J. K., Duffy, S. W., Baldwin, D. R., Brain, K. E., Devaraj, A., Eisen, T. et al. The UK Lung Cancer Screening Trial: a pilot randomised controlled trial of low-dose computed tomography screening for the early detection of lung cancer. Health Technol. Assess. 20, 1-146 (2016).

12. Grover, H., Ross, T. \& Fuller, E. Implementation of targeted screening for lung cancer in a high-risk population within routine NHS practice using low-dose computed tomography. Thorax 75, 348-350 (2020). 
13. NHS England. NHS to rollout lung cancer scanning trucks across the country. https://www.england.nhs.uk/2019/02/lung-trucks/ (2019).

14. National Cancer Programme. Targeted screening for lung cancer with low radiation dose computed tomography. Standard protocol prepared for the NHS England Targeted Lung Health Checks Programme. Version 1. (NHS, 2019).

15. US Preventive Services Task Force. Lung Cancer Screening Draft Recommendation Statement. https://www.uspreventiveservicestaskforce.org/uspstf/draftrecommendation/lung-cancer-screening-2020 (2020).

16. Kovalchik, S. A., Tammemagi, M., Berg, C. D., Caporaso, N. E., Riley, T. L., Korch, M. et al. Targeting of low-dose $C T$ screening according to the risk of lung-cancer death. N. Engl. J. Med. 369, 245-254 (2013).

17. Tammemägi, M. C., Katki, H. A., Hocking, W. G., Church, T. R., Caporaso, N., Kvale, P. A. et al. Selection criteria for lung-cancer screening. N. Engl. J. Med. 368, 728-736 (2013).

18. Katki, H. A., Kovalchik, S. A., Berg, C. D., Cheung, L. C. \& Chaturvedi, A. K. Development and validation of risk models to select ever-smokers for $\mathrm{CT}$ lung cancer screening. JAMA 315, 2300-2311 (2016).

19. Ten Haaf, K., Bastani, M., Cao, P., Jeon, J., Toumazis, I., Han, S. S. et al. A comparative modeling analysis of risk-based lung cancer screening strategies. J. Natl Cancer Inst. 112, 466-479 (2019).

20. Cassidy, A., Myles, J. P., van Tongeren, M., Page, R. D., Liloglou, T., Duffy, S. W. et al. The LLP risk model: an individual risk prediction model for lung cancer. Br. J. Cancer 98, 270-276 (2008).

21. Katki, H. A., Petito, L. C., Cheung, L. C., Jacobs, E., Jemal, A., Berg, C. D. et al. Implications of 9 risk prediction models for selecting ever-smokers for CT lungcancer screening. Ann. Intern. Med. 169, 10-19 (2018).

22. Ten Haaf, K., Jeon, J., Tammemägi, M. C., Han, S. S., Kong, C. Y., Plevritis, S. K. et al. Risk prediction models for selection of lung cancer screening candidates: a retrospective validation study. PLoS Med. 14, e1002277 (2017).

23. Li, K., Husing, A., Sookthai, D., Bergmann, M., Boeing, H., Becker, N. et al. Selecting high-risk individuals for lung cancer screening: a prospective evaluation of existing risk models and eligibility criteria in the German EPIC cohort. Cancer Prev. Res. 8, 777-785 (2015)

24. Weber, M., Yap, S., Goldsbury, D., Manners, D., Tammemagi, M., Marshall, H. et al. Identifying high risk individuals for targeted lung cancer screening: Independent validation of the PLCO m2012 risk prediction tool. Int. J. Cancer 141, 242-253 (2017).

25. Sudlow, C., Gallacher, J., Allen, N., Beral, V., Burton, P., Danesh, J. et al. UK Biobank: an open access resource for identifying the causes of a wide range of complex diseases of middle and old age. PLoS Med. 12, e1001779 (2015).

26. Riboli, E., Hunt, K. J., Slimani, N., Ferrari, P., Norat, T., Fahey, M. et al. European Prospective Investigation into Cancer and Nutrition (EPIC): study populations and data collection. Public Health Nutr. 5, 1113-1124 (2002).

27. Swerdlow, A. J., Jones, M. E., Schoemaker, M. J., Hemming, J., Thomas, D., Williamson, J. et al. The Breakthrough Generations Study: design of a long-term UK cohort study to investigate breast cancer aetiology. Br. J. Cancer 105, 911-917 (2011).

28. Bach, P. B., Kattan, M. W., Thornquist, M. D., Kris, M. G., Tate, R. C., Barnett, M. J. et al. Variations in lung cancer risk among smokers. J. Natl Cancer Inst. 95, 470-478 (2003).

29. Field, J. K., Vulkan, D., Davies, M. P. A., Duffy, S. W. \& Gabe, R. Liverpool Lung Project lung cancer risk stratification model: calibration and prospective validation. Thorax 76, 161-168 (2021).
30. Hoggart, C., Brennan, P., Tjonneland, A., Vogel, U., Overvad, K., Østergaard, J. N. et al. A risk model for lung cancer incidence. Cancer Prev. Res. 5, 834-846 (2012).

31. Landy, R., Cheung, L. C., Berg, C. D., Chaturvedi, A. K., Robbins, H. A. \& Katki, H. A Contemporary implications of U.S. Preventive Services Task Force and risk-based guidelines for lung cancer screening eligibility in the United States. Ann. Intern. Med. 171, 384 (2019).

32. Cheung, L. C., \& Katki, H. A. Icmodels R package. NCl Division of Cancer Epidemiology and Genetics: Tools and Resources. https://dceg.cancer.gov/tools/riskassessment/lcmodels (2018).

33. Fry, A., Littlejohns, T. J., Sudlow, C., Doherty, N., Adamska, L., Sprosen, T. et al. Comparison of sociodemographic and health-related characteristics of UK Biobank participants with those of the general population. Am. J. Epidemiol. 186, 1026-1034 (2017)

34. Haddad, D. N., Sandler, K. L., Henderson, L. M., Rivera, M. P. \& Aldrich, M. C. Disparities in lung cancer screening: a review. Ann. Am. Thorac. Soc. 17, 399-405 (2020).

35. Mazzone, P. J., Silvestri, G. A., Patel, S., Kanne, J. P., Kinsinger, L. S., Wiener, R. S. et al. Screening for lung cancer: CHEST guideline and expert panel report. Chest 153, 954-985 (2018).

36. Advani, S. \& Braithwaite, D. Optimizing selection of candidates for lung cancer screening: role of comorbidity, frailty and life expectancy. Transl. Lung Cancer Res. 8, S454-S459 (2019).

37. Cheung, L. C., Berg, C. D., Castle, P. E., Katki, H. A. \& Chaturvedi, A. K. Life-gainedbased versus risk-based selection of smokers for lung cancer screening. Ann. Intern. Med. 171, 623-632 (2019).

38. Yorkshire Cancer Research. Yorkshire Cancer Research announces UK's largest lung cancer screening trial. https://yorkshirecancerresearch.org.uk/news/ yorkshire-cancer-research-announces-uks-largest-lung-cancer-screening-trial (2017).

39. National Cancer Institute. Risk-based NLST Outcomes Tool (RNOT). https:// analysistools.nci.nih.gov/lungCancerScreening (2017).

40. Liverpool Lung Project. MyLungRisk. www.mylungrisk.org (2019).

41. Brock University. Lung cancer risk calculators. https://brocku.ca/lung-cancerscreening-and-risk-prediction/risk-calculators/ (2019).

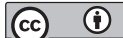

Open Access This article is licensed under a Creative Commons Attribution 4.0 International License, which permits use, sharing, adaptation, distribution and reproduction in any medium or format, as long as you give appropriate credit to the original author(s) and the source, provide a link to the Creative Commons license, and indicate if changes were made. The images or other third party material in this article are included in the article's Creative Commons license, unless indicated otherwise in a credit line to the material. If material is not included in the article's Creative Commons license and your intended use is not permitted by statutory regulation or exceeds the permitted use, you will need to obtain permission directly from the copyright holder. To view a copy of this license, visit http://creativecommons. org/licenses/by/4.0/.

(c) World Health Organization 2021, corrected publication 2021 